\title{
Inadequate diabetic care: global figures cry for preventive measures and personalized treatment
}

\author{
Babitha George • Melanie Cebioglu • \\ Kristina Yeghiazaryan
}

Received: 18 January 2010 / Accepted: 28 January 2010/Published online: 23 March 2010

(C) European Association for Predictive, Preventive and Personalised Medicine 2010

\begin{abstract}
Diabetes mellitus (DM) is a lifelong progressive disease. Currently there are more than 285 million DMaffected people worldwide. Globally the prevalence of diabetes continues to rise and is more pronounced in countries with large populations such as China, India and USA. Driving forces behind the epidemic are obesity, aging of the population and longer life expency. Prospective, the majority of diabetic population will be in their six to eight decades of life with the implication of more females than males. Severe micro- and macrovascular complications associated with diabetes lead to a highly increased morbidity and mortality. Therefore, DM is projected to be one of the leading health problems of 21 st centuary. Urgent measures are required to reduce the diabetic burden. Thus advanced predictive diagnostic tools and personalized treatment strategies in (pre)diabetic care are critical and should exert beneficial impact on public health.
\end{abstract}

Keywords Diabetes epidemic scale · Economical burden . Morbidity mortality · Healthcare-providers · Policy-makers . Related pathologies

\section{Global prevalence of Diabetes mellitus}

Diabetes mellitus (DM) is a lifelong progressive disease. It is classified as a group of heterogeneous metabolic disorders characterized by hyperglycemia as a common feature. The vast majority cases of DM fall mainly into two

\footnotetext{
B. George $\cdot$ M. Cebioglu $\cdot$ K. Yeghiazaryan $(\triangle)$

Division of Molecular/Experimental Radiology, Department

of Radiology, Rheinische Friedrich-Wilhelms-University of Bonn,

Sigmund-Freud-Str. 25,

53105 Bonn, Germany

e-mail: kristina.yeghiazaryan@ukb.uni-bonn.de
}

broad etiopathogenetic categories, classified as type 1 and type $2 \mathrm{DM}$. Type $1 \mathrm{DM}$ is induced by beta cell destruction, whereas pancreatic beta cell dysfunction and insulin resistance are hallmark features of type $2 \mathrm{DM}$.

A number of factors are known to impact the disease development. Genetic predisposition, is well-acknowledged for both types of DM [1]. Further factors can be summarized as environmental influence, life style, increased stress, nutritional behaviors, such as frequent fast food consumption, and low physical activity. As a consequence, the number of diabetic patients is continuously increasing worldwide.

The prevalence of DM has already achieved epidemic proportion with more than 285 million patients worldwide [2]. Statistically, DM-affected patients comprise almost $6.4 \%$ of the world's population [3]. The incidence of type $1 \mathrm{DM}$ accounts for $5-10 \%$ of all new DM diagnoses, while the dominating type $2 \mathrm{DM}$ accounts for $90-95 \%$ of all cases worldwide [4].

Diabetes prevalence will more than double over the next 30 years and is projected to rise from $4.4 \%$ in 2000 to $7.7 \%$ in 2030. Consequently, the worldwide prevalence of diabetes amoung adults (20-79 years of age) is expected to increase up to about 439 million by the year 2030 [3]. The projections for DM prevalence are becoming more pessimistic year by year (see Fig. 1).

Main contributing factors can be summarized as aging of the population, urbanization, increasing prevalence of obesity and low physical activity etc. [5-9]. The increased prevalence of DM is predicted to occur virtually for every nation, while the greatest increase is expected in developing countries $[10,13]$. In developed countries, the prevalence of DM has been shown to be similar in urban and rural areas. In contrast, significantly higher DM prevalence occurs in the urban population of developing countries, 
Fig. 1 Global prevalence of diabetes mellitus (DM) for the years 1995-2030. Total DMaffected population is projected to rise from 285 million in 2010 to 439 million in 2030. Worldwide, a strong increase in diabetic burden is predicted, particularly starting from the year 2020 [3, 5, 8-12]

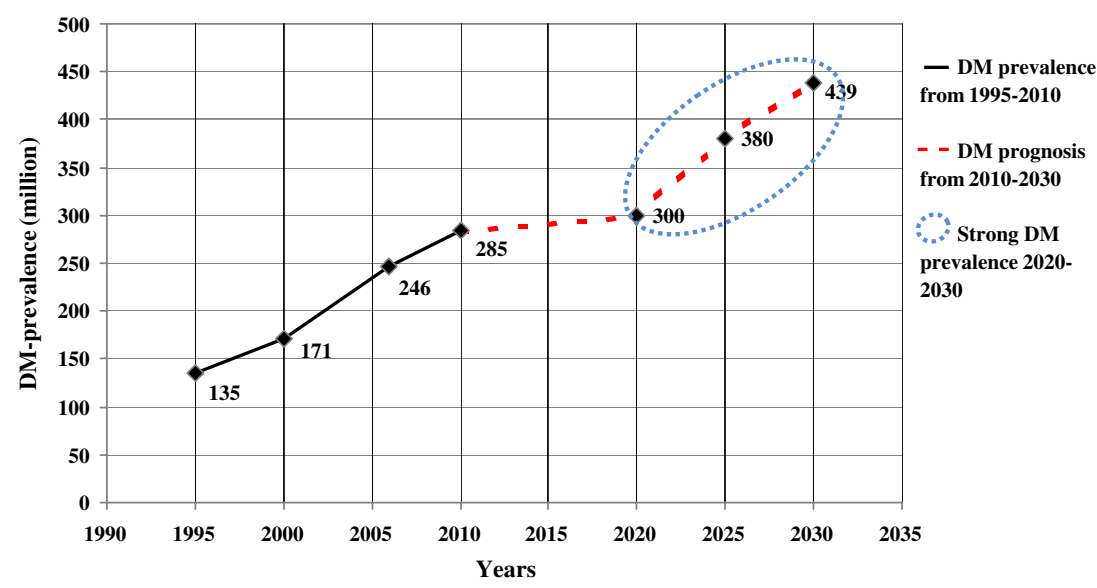

which may relate to factors such as unbalanced diet, obesity, decreased physical activity and stress [10]. The prospective estimates are that from 2010 to 2030, there will be an increase of $69 \%$ in the number of adult diabetic pateints for the developing countries, while for developed countries it is expected to increase by $20 \%$ [3].

According to NIDDK (National Institute of Diabetes and Digestive and Kidney Diseases), people of AfricanCaribbean, Asian, and Hispanic origin are more frequently affected by the disease [14]. Furthermore, prognosis for the year 2030 is demonstrating that countries with high population numbers, such as China, India and USA, will have a bigger increase in both absolute number and percentage of DM cases in population (see Fig. 2). Worldwide, India has the highest absolute number registered for diabetic patients in 2010. The estimated DM population is 50.8 million, while the projection for the future is rising up to 87 million DM-affected people for 2030 [3]. In Europe Germany is listed among top ten countries having the highest estimated number of diabetics in the world $[15,16]$.

\section{Impact of aging, education and socioeconomic status on DM prevalence}

Aging is one of the major factors promoting the epidemic scale of DM [17]. Currently diabetes is seen to be highly prevalenced in the two age groups of 40-59 and 60-79 years with 131 and 108 million affected individuals, respectively (see Fig. 3). By 2030, it is predicted that there will be 188 million people with diabetes aged 40-59 years and considerably even more people in the age group over 60 years old, estimated to be about 196 million [2]. In addition, both industrialization and globalization affect the rising prevalence of diabetes in the younger age categories including teenagers [18].

In most countries, a lower educational level was associated with higher prevalence of diabetes compared to better educated groups. In western countries, the risk for developing diabetes is strongly linked to a low socioeconomic status. Most of the studies have shown that people with higher socioeconomic position have a low prevalence of diabetes, while people with low socioeconomic status
Fig. 2 Top six countries with highest DM-affected population between 20-79 years of age in 2010 and 2030 [2]. India has the largest DM prevalence, with the growth rate percentage of $71.3 \%$, followed by China (44.9\%), USA (34.3\%) and Russia (7.3\%). For Germany and Japan the prevalence data for the year 2030 are currently not available

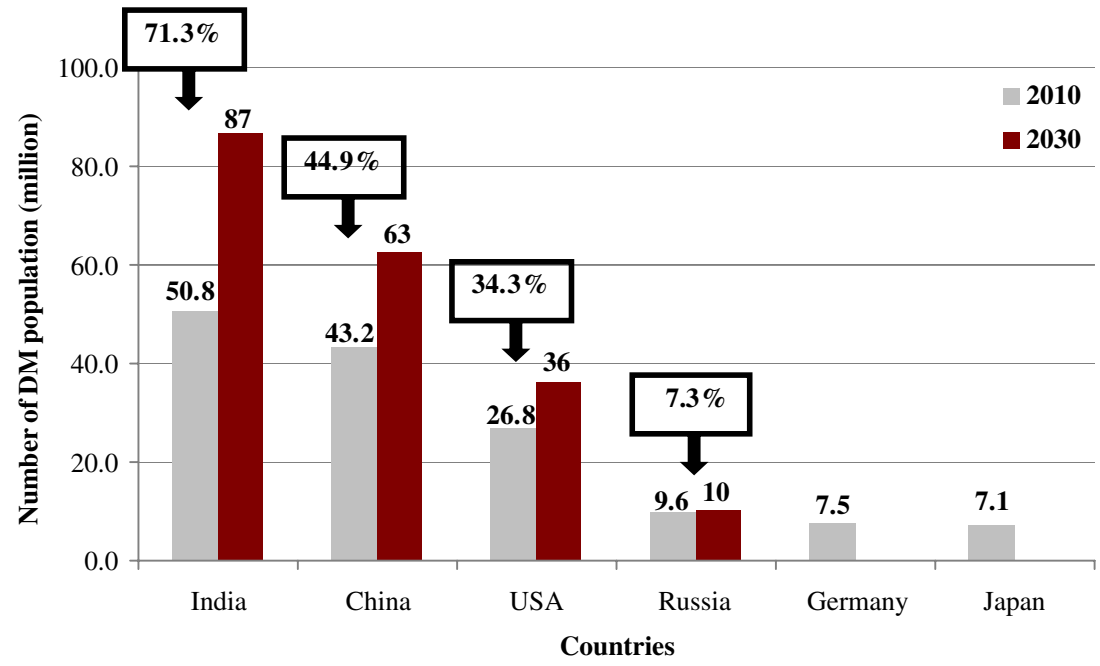




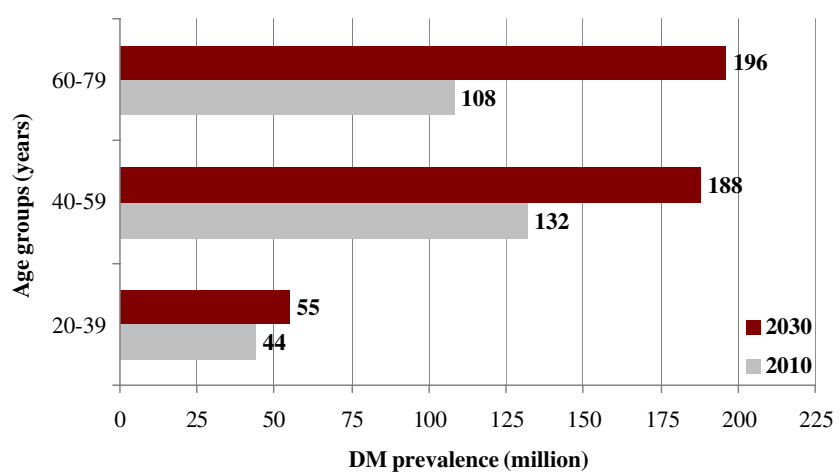

Fig. 3 Global DM prevalence in different age groups for the years 2010 and 2030 [2]. Current DM prevalence is likely to be largest among the age group of 40-59 years with 132 million people, followed by the age group of 60-79 years with 108 million. In 2030, an increase is expected in all age groups, with greatest number of people in the 60-79 years of life. Estimates indicate an increase of 88 million and 56 million for the age groups of 60-79 years and 4059 years, respectively

have a higher prevalence of the disease [9, 19-22]. Further, the influence of socioeconomic factors on DM prevalence has been shown to be higher among women compared to men.

\section{Gender aspect in DM-Prevalence}

Eventhough global prevalence of Diabetes mellitus is similar in men and women (see Fig. 4), it has been demonstrated that age plays a major role in gender prevalence. In the age above 65 years, diabetes tends to be more frequent in women than men $[10,23]$.

\section{Prevalence of DM-associated complications and mortality}

Chronic complications, secondary to diabetes are affecting many organ systems, being responsible for a considerably shortened life-expectancy and sudden death (see Fig. 5a and b). Specific complications of diabetes depend on many factors including the duration of the metabolic derangements.

Microvascular complications, due to damage to small blood vessels, are involved in the pathomechanisms of all chronic DM-related pathologies. Diabetic neuropathy is the leading DM-associated complication. Peripheral sensory neuropathy is the most common form of diabetic neuropathy affecting $40-50 \%$ of patients with diabetes [27]. It mainly affects the feet, leading to foot lesions, numbness and foot/toe amputation. Diabetic retinopathy, the earliest manifestation of organ damage developed in DM population, is the second most common complication (see Fig. 5a). In diabetic patients, nephropathy is the main risk factor for all cardiovascular mortalities [28]. Recent studies in the United States implicate chronic nephropathy as the leading cause of renal failure. It is affecting also up to $40 \%$ of patients with diabetes worldwide [29].

Macrovascular complications, which relate to the heart and large blood vessels, are peripheral vascular diseases, coronary artery diseases, arthrosclerosis and stroke [30].

Cancer provocation in diabetes Recent research shows that diabetic patients are generally predisposed to cancer $[31,32]$.

Mortality Worldwide every $10 \mathrm{~s}$ a person dies due to a diabetes-related pathology. Furthermore, DM is documented to be the fourth leading cause of death. In the year 2010, the global mortality attributed to diabetes was estimated to be 3.96 million, showing an increase of 5.5\% when compared to the mortality in the year 2007 [23]. The percentage of all death in the diabetic population between 20-79 years of age was lowest (6\%) in the poorest African countries and highest in the North American region (15.7\%) (see Fig. 6). Doubling of the population will be the main reason for the global burden of diabetes in the next 25 years [33]. The highest number of deaths due to diabetes is projected to occur in countries with large populations such as China, India, USA and the Russian Federation. More women than men are expected to die from diabetes-related complications, thus diabetes contributes significantly to female mortality [34].

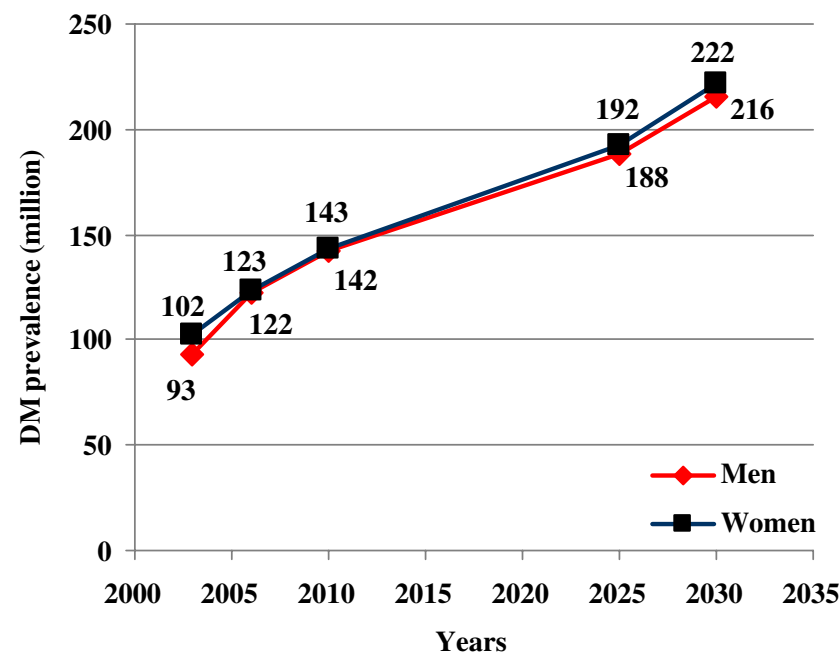

Fig. 4 Global prevalence of diabetes in men and women aged between 20-79 years, estimated for the years 2003-2030 [2, 23, 24]. Currently, there are $6.6 \%$ people with diabetes mellitus worldwide; 143 million of them are women and 142 million are men. Prospectively, the prevalence of DM will continue to increase. For 2030 , it is expected that there will be about 6 million more women than men with diabetes (222 million women versus 216 million men) 


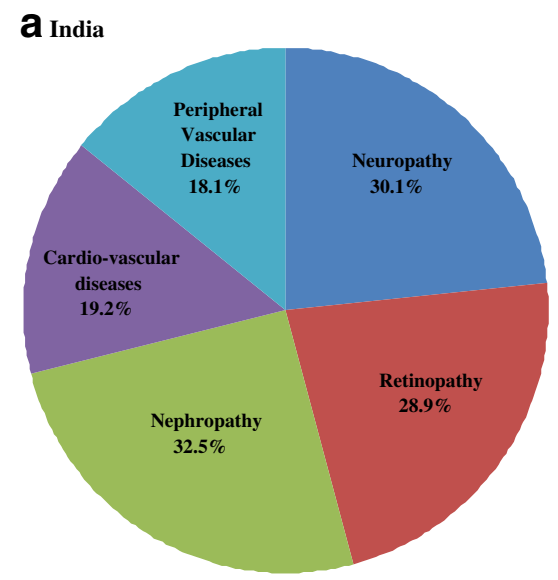

Fig. 5 a. Prevalence of type 2 DM micro- and macrovascular complications, in percentage, for Indian population during the year 2000. Among microvascular complications, nephropathy $(32.5 \%)$ is the most frequent followed by neuropathy and retinopathy with $30.1 \%$ and $28.9 \%$, respectively. Among macrovascular complications, cardiovascular complications are more common and hypertension is significantly associated with all complications [25]. b. Prevalence of

The percentage of excess deaths due to diabetes was highest in people 55-59 years old, currently having the largest effect in South-East Asia (see Fig. 7). When comparing similar age groups of DM-affected versus general population, the annual mortality rate has been documented to be two-fold higher in diabetic patients. Each

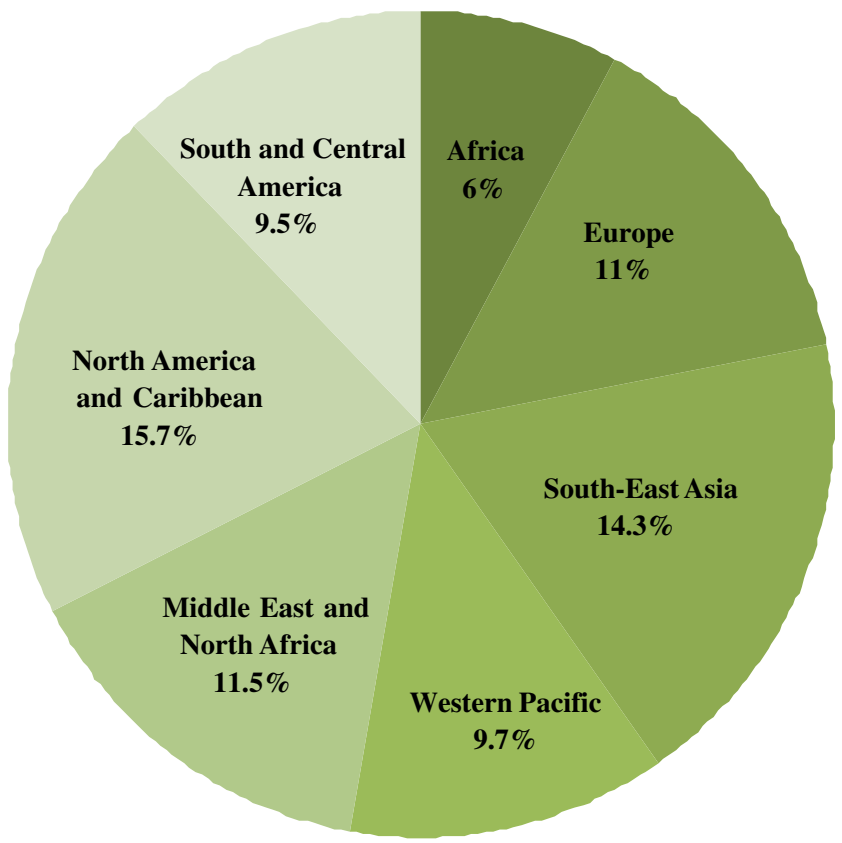

Fig. 6 Worldwide documented DM-associated mortality for the population aged between 20-79 years in contest of regional differences as updated for 2010 [23]. Diabetes accounted for over 1 in 20 deaths. Total percentage of diabetic mortality is very high particularly in North America (15.7\%) and South-East Asia (14.3\%), both regions with large populations

\section{b uSA}

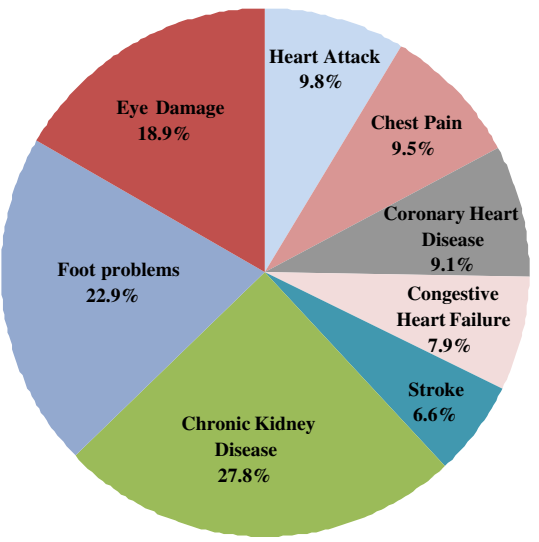

type 2 DM complications, in percentage, for USA, from NHANES (National Health and Nutrition Examination Survey) for the years 1999-2004 [26]. Nephropathy is one of the major microvascular complications, with the prevalence of $25 \%$ followed by foot problems and eye damage. Heart disease are prevalent common among macrovascular complications

year nearly 3 million people die because of diabetes, and two thirds of them die in developing countries [33].

\section{Conclusions}

According to the information provided above, we conclude:

- DM micro- and macrovascular complications are one of the leading health problems of 21 st century.

- Over the next 20 years, DM-affected population is likely to be increase by $50 \%$.

- Although prevalence of diabetes continues to rise in every nation, the highest increase is expected to occur in countries with large populations - China, India and USA.

- Urban population in developing countries is projected to double which will likely lead to increased diabetic prevalence.

- Most important demographic change in DM prevalence across the world appears to be the increase in older population (age above 60 years), as a consequence of longer life span.

- DM is more frequent in females than males.

- The progressive prevalence of DM will lead to a very high economic burden.

- Severe complications lead to highly increased morbidity and mortality among DM patients. Global excess mortality due to diabetes is more pronounced in developing countries and individuals with low socioeconomic status.

Urgent measures should be considered:

- Early prevention of DM in childhood and adults

- Improved physical activity, individual nutrition and lifestyle 


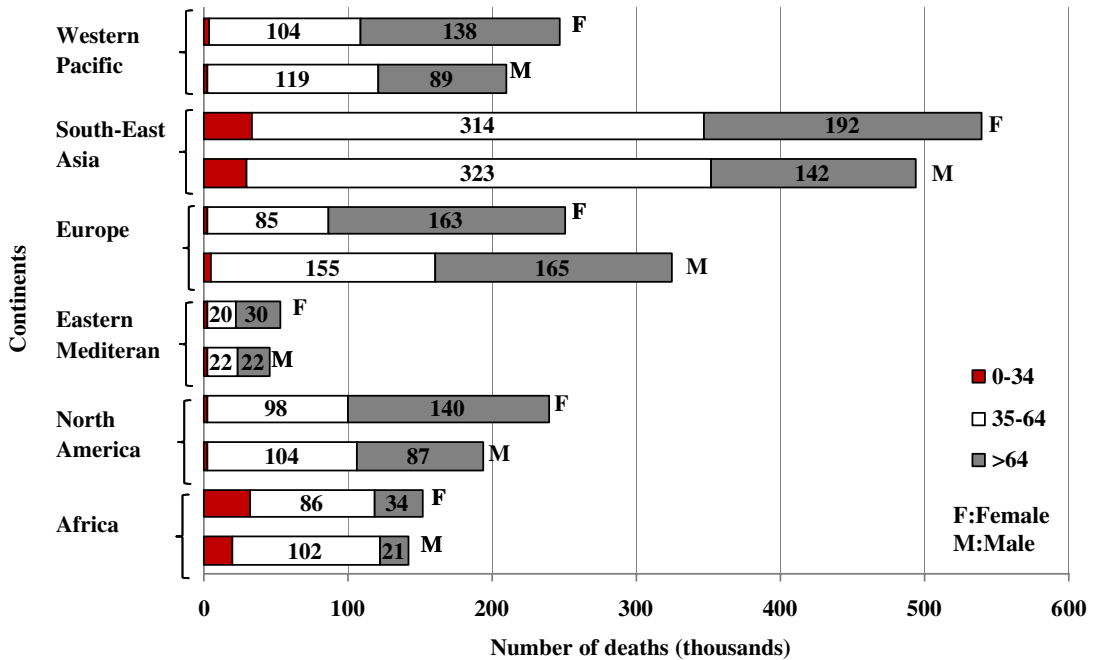

Fig. 7 Number of deaths (in thousands) attributable to diabetes by age groups and gender. Global mortality was lowest in the Eastern Mediteran and Africa regions, while the highest numbers were documented in South-East Asia and America. DM-associated deaths are particularly increased for the age group of 35-64 years, in regions where the prevalence of diabetes is very high in younger ages (South East Asia, America and Africa). In Europe, DM mortality tends to be

- Application of advanced predictive diagnostics to preventively treat individuals at high risk

- Personalized treatment in (pre)diabetes

\section{References}

1. Mehers KL, Gillespie KM. The genetic basis for type 1 diabetes. Br Med Bull. 2008;88:115-29.

2. International Diabetes Federation. The Diabetes Atlas (Fourth edition) International Diabetes Federation:Brussels. http://www. diabetesatlas.org/downloads; 2010.

3. Shaw JE, Sicree RA, Zimmet PZ. Global estimates of the prevalence of diabetes for 2010 and 2030. Diabetes Res Clin Pract (Epub ahead of print). PMID: 19896746; 2009.

4. Centers for Disease Control and Prevention. National diabetes fact sheet: general information and national estimates on diabetes in the United States, Atlanta, GA: U.S. Department of Health and Human Services, Centers for Disease Control and Prevention, 2008. http://www.cdc.gov/diabetes/pubs/pdf/ndfs 2007.pdf; 2007.

5. Seidell JC. Obesity, insulin resistance and diabetes-a worldwide epidemic. Br J Nutr. 2000;83:S5-8.

6. Scheen AJ. Pathophysiology of type 2 diabetes. Acta Clin Belg. 2003;58:335-41.

7. Mokdad AH, Ford ES, Bowman BA, et al. Prevalence of obesity, diabetes, and obesity-related health risk factors, 2001. JAMA. 2003;289:76-9.

8. Magliano DJ, Shaw JE, Shortreed SM, et al. Lifetime risk and projected population prevalence of diabetes. Diabetologia. 2008;51:2179-86.

9. Rifat-uz-Zaman. High prevalence of Diabetes mellitus and promoting factors among human urban population of Bahawalpur-district, Pakistan: cross-sectional study. Res J Med Sci. 2009;3:62-9. more frequent in men than in women. On the other hand, for all other regions, the prevalence of DM mortality is higher in female population, mainly in the age group older than 64 years. Among the younger age group of $0-34$ years, the numbers of deaths were high in South-East Asia (29.1 thousand men and 33.1 thousand women) and Africa (20 thousand men and 32.2 thousand women) [33]

10. Wild S, Roglic G, Green A, et al. Global prevalence of diabetes: estimates for the year 2000 and projections for 2030. Diabetes Care. 2004;27:1047-53

11. World Health Organization (2009) Fact sheet $N^{\circ} 312$. http://www. who.int/mediacentre/factsheets/fs $312 / \mathrm{en} /$

12. International Diabetes Federation. The Diabetes Atlas. 3rd edn. International Diabetes Federation:Brussels. http://www. diabetesatlas.org/; 2006.

13. Hossain P, Kawar B, El Nahas M. Obesity and diabetes in the developing world-a growing challenge. $N$ Engl J Med. 2007;356:213-5.

14. Chan JC, Malik V, Jia W, et al. Diabetes in Asia: epidemiology, risk factors, and pathophysiology. JAMA. 2009;301:2129-40.

15. Rathmann W, Giani G. Global prevalence of diabetes: estimates for the year 2000 and projections for 2030. Diabetes Care. 2004;27:2568-9.

16. Frese T, Sandholzer H, Voigt S, et al. Epidemiology of diabetes mellitus in German general practitioners' consultation-results of the SESAM 2-study. Exp Clin Endocrinol Diabetes. 2008;116:326-8.

17. Kolb H, Mandrup-Poulsen T. The global diabetes epidemic as a consequence of lifestyle-induced low-grade inflammation. Diabetologia [Epub ahead of print]. PMID: 19890624; 2009.

18. Zimmet P, Alberti KG, Shaw J. Global and societal implications of the diabetes epidemic. Nature. 2001;414:782-7.

19. Espelt A, Borrell C, Roskam AJ. Socioeconomic inequalities in diabetes mellitus across Europe at the beginning of the 21st century. Diabetologia. 2008;51:1971-9.

20. Dalstra JA, Kunst AE, Borrell C, et al. Socioeconomic differences in the prevalence of common chronic diseases: an overview of eight European countries. Int J Epidemiol. 2005;34:316-26.

21. Connolly V, Unwin N, Sherriff P, et al. Diabetes prevalence and socioeconomic status: a population based study showing increased prevalence of type 2 diabetes mellitus in deprived areas. J Epidemiol Community Health. 2000;54:173-7.

22. Rabi DM, Edwards AL, Southern DA, et al. Association of socioeconomic status with diabetes prevalence and utilization of diabetes care services. BMC Health Serv Res. 2006;6:124. 
23. Roglic G, Unwin N. Mortality attributable to diabetes: Estimates for the year 2010. Diabetes Res Clin Pract (Epub ahead of print). PMID: 19914728; 2009.

24. International Diabetes Federation. The Diabetes Atlas, 2nd edn. International Diabetes Federation:Brussels. http://www.idf.org/; 2003.

25. Agrawal RP, Ranka M, Beniwal R et al. Prevalence of micro and macro vascular complications in type 2 diabetes and their riskfactors. Int J Diab Dev Countries. 2004; 24.

26. National Health and Nutrition Examination Survey (NHANES). Firstof-its-Kind National Report Reveals Estimated High Prevalence and Heavy Cost of Type 2 Diabetes Complications in America. http:// www.prnewswire.com/news-releases/first-of-its-kind-national-reportreveals-estimated-high-prevalence-and-heavy-cost-of-type-2-diabetescomplications-in-america-58000707.html; 1999-2004.

27. Richardson LC, Pollack LA. Therapy insight: Influence of type 2 diabetes on the development, treatment and outcomes of cancer. Nat Clin Pract Oncol. 2005;2:48-53.
28. Noh H, King GL. The role of protein kinase C activation in diabetic nephropathy. Kidney Int. 2007;106:S49-53.

29. Shinada M, Akdeniz A, Panagiotopoulos S, et al. Proteolysis of insulinlike growth factor-binding protein-3 is increased in urine from patients with diabetic nephropathy. J Clin Endocrinol Metab. 2000;85:1163-9.

30. Ban CR, Twigg SM. Fibrosis in diabetes complications: pathogenic mechanisms and circulating and urinary markers. Vasc Health Risk Manag. 2008;4:575-96.

31. Golubnitschaja O. Advanced diabetes care: three levels of prediction, prevention \& personalized treatment. Curr Diabetes Rev. 2010;6:42-51.

32. Cebioglu M, Schild HH, Golubnitschaja O. Diabetes mellitus as a risk factor for cancer: stress or viral etiology? Infect Disord Drug Targets. 2008;8:76-87.

33. Roglic G, Unwin N, Bennett PH, et al. The burden of mortality attributable to diabetes: realistic estimates for the year 2000 . Diabetes Care. 2005;28:2130-5.

34. Roglic G. Diabetes in women: the global perspective. Int $\mathrm{J}$ Gynaecol Obstet. 2009;104:S11-3. 